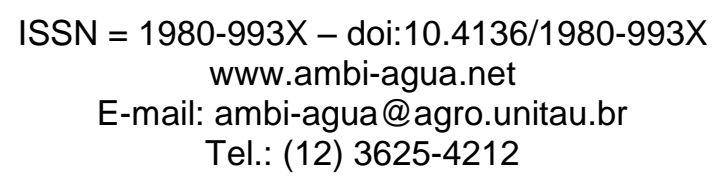

\title{
Remoção dos corantes Reactive Blue 21 e Direct Red 80 utilizando resíduos de sementes de Mabea fistulifera Mart. como biossorvente
}

\author{
(http://dx.doi.org/10.4136/ambi-agua.854)
}

\author{
Julieta de Jesus da Silveira Neta ${ }^{1,2}$; Carlos Juliano da Silva ${ }^{2}$; \\ Guilherme Costa Moreira ${ }^{2}$; César Reis ${ }^{2}$; Efraim Lázaro Reis ${ }^{2}$ \\ ${ }^{1}$ Embrapa Amazônia Oriental, Belém, Pará \\ e-mail: julieta_neta@yahoo.com.br; julieta@cpatu.embrapa.br \\ ${ }^{2}$ Universidade Federal de Viçosa (UFV), Viçosa, Minas Gerais \\ e-mail: carlosjuliano07@yahoo.com.br; songuilherme@yahoo.com.br; \\ cesar@ufv.br; efraim@ufv.br
}

\section{RESUMO}

Neste estudo empregou-se resíduos de Mabea fistulifera Mart. como bioadsorvente para a remoção dos corantes Reactive Blue 21 (RB 21) e Direct Red 80 (DR 80) em soluções aquosas e em amostras de efluentes reais fortificados com estes corantes. Foram investigados os ensaios da influência do $\mathrm{pH}$, cinéticos e adsortivos para a remoção dos corantes por meio de uma série de experimentos de batelada. O bioadsorvente exibiu máxima adsorção em $\mathrm{pH}=2,0$ para ambos os corantes. O tempo de equilíbrio da adsorção foi estabelecido em 300 minutos para o DR 80 e 120 minutos para o RB 21. O modelo de Langmuir descreveu com maior fidelidade o comportamento do sistema sortivo, apresentando coeficiente de determinação $\left(\mathrm{R}^{2}\right)$ superior a 0,98 . Por meio da Isoterma de Langmuir, foi possível obter a capacidade máxima de adsorção dos corantes Direct Red 80 e Reactive Blue 21 pelo bioadsorvente sendo encontrados os valores de 4,92 $\mathrm{mg} \mathrm{g}^{-1}$ e $11,13 \mathrm{mg} \mathrm{g}^{-1}$, respectivamente. O modelo cinético de pseudo-segunda ordem melhor descreveu o processo de adsorção do corante RB 21 sob o bioadsorvente, embora a difusão intra-partícula também esteja envolvida no mecanismo de adsorção. Já para o corante DR 80 o modelo de difusão de Morris e Weber sugeriu que a difusão intra-partícula é predominante em todo o processo de adsorção. Depois de otimizadas as condições adsortivas, o bioadsorvente foi empregado a amostras de efluentes têxteis reais fortificadas com soluções aquosas dos corantes, obtendo-se $85 \%$ de remoção do RB 21 e $94 \%$ de remoção do DR 80 na matriz do efluente.

Palavras-chave: adsorção; Mabea fistulifera Mart.; bioadsorvente; corante.

\section{Removal of the Reactive Blue 21 and Direct Red 80 dyes using seed residue of Mabea fistulifera Mart. as biosorbent}

\section{ABSTRACT}

Residues of Mabea fistulifera Mart. were used in this study as a bioadsorbent for the removal of the Reactive Blue 21 (RB 21) and Direct Red 80 (DR 80) dyes from aqueous solutions and samples of real effluents enriched with these dyes. The influence of $\mathrm{pH}$, kinetic, and adsorptive parameters for the removal these dyes were investigated by conducting a series of batch experiments. The maximum adsorption using this bioadsorbent was observed at $\mathrm{pH}=2.0$ for both dyes. The time of adsorption equilibrium was established at 300 minutes for DR 80 and 120 minutes for RB 21. The Langmuir model stood out because it described the 
behavior of the sorptive system with higher fidelity than the Freundlich model showing the coefficient of determination $\left(\mathrm{R}^{2}\right)$ above 0.98 . It was possible to obtain the maximum adsorption capacity of the bioadsorbent, for the Direct Red 80 and Reactive Blue 21 dyes, using the Langmuir Isotherm; these values were determined as $4.92 \mathrm{mg} \mathrm{g}^{-1}$ and $11.13 \mathrm{mg} \mathrm{g}^{-1}$, respectively. The pseudo-second order kinetic model best described the adsorption process of the RB 21 dye by the bioadsorbent, however, the intra-particle diffusion is also involved in the mechanism of adsorption. The Morris and Weber diffusion model suggested that the intraparticle diffusion is prevalent throughout the process of adsorption for the DR 80 dye. The bioadsorbent was employed on real textile effluent samples enriched with aqueous solutions of these dyes after the adsorptive conditions were optimized; $85 \%$ RB 21 and $94 \%$ DR 80 removal rates were obtained from the effluent's matrix.

Keywords: adsorption; Mabea fistulifera Mart.; biosorbent; dye.

\section{INTRODUÇÃO}

As indústrias são os principais responsáveis pela geração de efluentes contendo compostos fenólicos (Froehner et al., 2009), metais pesados (Ghorbani et al., 2008; Lohani et al., 2008) e corantes (Baek et al., 2010; Brito et al., 2010; Saha, 2010). Estima-se que são utilizados mundialmente mais de 10.000 tipos diferentes de corantes e pigmentos. $\mathrm{Na}$ indústria têxtil, $15 \%$ dos corantes utilizados são perdidos durante o processo de tingimento de tecidos gerando efluentes (Parshetti et al., 2010). Quando tais efluentes são descartados indevidamente em corpos hídricos, os corantes mesmo em concentrações baixas são prejudiciais (Park et al., 2007). Se não forem tratados adequadamente antes de lançados em águas naturais, os efluentes provenientes da indústria de corantes ou de processos envolvendo tintura têxtil podem modificar o ecossistema ou atingir a saúde da população. Alguns corantes como os pré-metalizados, chegam a liberar substâncias tóxicas com altas concentrações de metais pesados. Outros podem ser acumulados por plantas e, consequentemente, passar para a cadeia alimentar, contaminando outros organismos (Zanoni e Carneiro, 2001).

De maneira geral, o processo mais comum utilizado na remoção de corantes de efluentes industriais é a adsorção. Sua maior aplicação industrial está associada ao baixo custo, flexibilidade e simplicidade do processo, facilidade de operação se comparada a outras técnicas de tratamento. Além disso, a adsorção não resulta na formação de substâncias prejudiciais ao meio ambiente, não utiliza solventes orgânicos, bem como pode permitir o reaproveitamento do adsorvente (Achak et al., 2009).

Atualmente o material que apresenta maior capacidade de adsorção, sendo amplamente utilizado para o tratamento de efluentes, é o carvão ativado. Entretanto, devido às perdas durante o processo de recuperação do adsorvente, sua utilização torna-se onerosa (Aktaş e Çeçen, 2007; Ozkaya, 2006). Neste sentido existe um crescente interesse pela busca de materiais alternativos de baixo custo que possam ser utilizados em substituição ao carvão ativado como adsorventes para a eliminação de corantes têxteis, tais como: casca de banana (Achak et al., 2009), casca de nozes (Brito et al., 2010), folhas de Neem (Immich et al., 2009), pó de folhas de abacaxi (Weng et al., 2009), casca de cevada (Robinson et al., 2002), bagaço de cana-de-açucar (Santos et al., 2011), sementes de Moringa oleifera (Viera et al., 2009) e o resíduo de semente de Mabea fistulifera Mart. alvo de nosso estudo. A Mabea pertence à família Euphorbiaceae, é uma planta nativa amplamente encontrada em áreas de transição de Mata para Cerrado, nos estados de Minas Gerais, Rio de Janeiro e São Paulo, muito utilizada para recuperação de áreas degradadas por indústrias de mineração. Árvore de alta importância ambiental e econômica, pois produz pólen e néctar em abundância, e sua semente produz uma quantidade de óleo de aproximadamente $40 \%$ podendo assim utilizada para a produção de 
biodiesel (Pereira, 2007). É popularmente conhecida como canudo-de-pito, sendo considerada uma planta de pequeno porte, com em média 7 metros de altura (Lorenzi, 2000).

Este trabalho visa estudar a eficiência de resíduos de semente de Mabea fistulifera mart. na remoção dos corantes Reactive Blue 21 (RB 21) e Direct Red 80 (DR 80) presentes em efluentes têxteis, bem como verificar a influência de diferentes parâmetros no processo de remoção da cor.

\section{MATERIAIS E MÉTODO}

\subsection{Aparelhagem}

As medidas de absorbância das soluções aquosas dos corantes Direct Red 80 (DR 80) e Reactive Blue 21 (RB 21) foram registradas em um espectrofotômetro (HITACHI, modelo U2000), utilizando-se cubetas de quartzo de $1 \mathrm{~cm}$ de caminho ótico e água deionizada como referência.

\subsection{Preparo da solução estoque de corantes}

Os corantes comerciais Reactive Blue 21 e Direct Red 80 foram cedidos pela Companhia Industrial Itabira do Campo (Itabirito, MG). Informações relevantes sobre as propriedades destes corantes estão dispostos na Tabela 1. As soluções estoque de cada um dos corantes

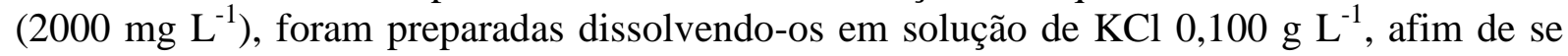
manter a força iônica do meio constante.

Tabela 1. Propriedades do DR 80 e RB 21.

\begin{tabular}{l|cc}
\hline \multicolumn{1}{c|}{ Propriedades } & Direct Red 80 & Reactive Blue 21 \\
\hline$\lambda_{\text {máx }}(\mathrm{nm})$ & 537 & 631 \\
Tipo & Direto & Reativo \\
Peso molecular $(\mathrm{g})$ & $1373,07 \mathrm{~g}$ & $1159,62 \mathrm{~g}$ \\
Fórmula molecular & $\mathrm{C}_{45} \mathrm{H}_{26} \mathrm{~N}_{10} \mathrm{Na}_{6} \mathrm{O}_{21} \mathrm{~S}_{6}$ & $\mathrm{C}_{40} \mathrm{H}_{26} \mathrm{CuN}_{10} \mathrm{O}_{16} \mathrm{~S}_{6}$ \\
Número no Color & 35780 & $12236-86-1$ \\
Index (C.I) & & \\
\hline
\end{tabular}

\subsection{Preparo do adsorvente}

A colheita das sementes da Mabea fistulifera Mart (canudo-de-pito) foi realizada no município de Viçosa MG, antes de seu período de maturação das mesmas. Posteriormente a essa etapa, as sementes foram colocadas sob o sol e cobertas com uma tela, com objetivo de se esperar a deiscência.

O óleo presente nas sementes de Mabea foi extraído e o resíduo das sementes gerado após a extração, foi lavado abundantemente com água destilada. Em seguida, foi conduzido à estufa para secagem a $60{ }^{\circ} \mathrm{C}$ durante seis horas, e peneirado de forma que sua granulometria se mantivesse em 65 mesh $(212 \mu \mathrm{m}$ de diâmetro). Depois de secos e peneirados, os resíduos das sementes foram utilizados para remoção de corantes por processos de adsorção.

\subsection{Caracterização do adsorvente}

A espectroscopia no infravermelho com transformada de Fourier foi utilizada para identificar os principais grupos funcionais, presentes na semente de Mabea. Os espectros do adsorvente foram registrados em espectrofotômetro infravermelho Perkin Elmer - FT - IR 1000, situados na faixa de 400 a $4000 \mathrm{~cm}^{-1}$, a partir de pastilhas de $\mathrm{KBr}$ preparadas com 2,00 mg de amostra. 
Para a determinação do ponto de carga zero (PCZ) do adsorvente, empregaram-se dois tubos de centrífuga, contendo 0, $500 \mathrm{~g}$ dos resíduos da semente. Em cada um dos tubos, foi adicionado $20 \mathrm{~mL}$ de solução de $\mathrm{NaCl}$ em concentrações de 0,1 e $0,015 \mathrm{~mol} \mathrm{~L}^{-1}$. Após a adição das soluções, as misturas foram agitadas durante 30 minutos e mantidas em contato por 72 horas (Egreja Filho et al., 2004).

A amostra do material adsorvente foi submetida a um processo de metalização por meio de recobrimento com ouro utilizando-se um metalizador (Sputtering-Balzeis, modelo FDV010). A visualização desta superfície foi conduzida em um microscópio eletrônico de varredura LEO modelo VP1430, pertencente ao Núcleo de Microscópia e Microanálise (CCBUFV).

\subsection{Ensaios adsortivos em batelada}

\subsubsection{Influência do pH na adsorção}

$\mathrm{O}$ ensaio para avaliação da influência do $\mathrm{pH}$ foi realizado em triplicata abrangendo a faixa de 2,0 a 9,0. Foram adicionados $0,080 \mathrm{~g}$ do adsorvente em tubos de centrífuga e 15,00 $\mathrm{mL}$ de soluções com $\mathrm{pH}$ devidamente ajustados de acordo com a faixa de estudo. $\mathrm{O} \mathrm{pH}$ foi monitorado até atingir sua estabilização, posteriormente os tubos foram centrifugados, o sobrenadante descartado e então foram adicionados $15,00 \mathrm{~mL}$ de solução do corante Reactive blue 21 na concentração de $30 \mathrm{mg} \mathrm{L}^{-1}$. O mesmo procedimento foi realizado para o corante Direct Red 80. Os tubos foram agitados por 540 minutos, a $25{ }^{0} \mathrm{C}$. Finalmente, as determinações quantitativas do corante remanescente no sobrenadante foram realizadas por meio de medidas de espectrometria de absorção UV/Vis.

\subsubsection{Estudos cinéticos de adsorção}

$\mathrm{O}$ ensaio cinético de adsorção foi realizado em $\mathrm{pH}$ ótimo $(\mathrm{pH}=2)$, a temperatura de $25{ }^{0} \mathrm{C}$. O estudo foi conduzido em recipiente de $250 \mathrm{~mL}$ devidamente fechado, sendo adicionados, 0,800 g de resíduos da semente de Mabea e $150 \mathrm{~mL}$ da solução dos corantes com concentração $30 \mathrm{mg} \mathrm{L}^{-1}$.

Em intervalos pré-determinados de tempo, foram coletadas alíquotas de 1,00 mL com utilização de uma seringa de vidro acoplada a um sistema filtrante contendo membrana Durapore $0,45 \mu \mathrm{m}$. As leituras das absorvâncias referentes às concentrações dos corantes foram determinadas em espectrofotômetro UV/Vis.

Os modelos de pseudo primeira ordem (Chen et al., 2011), de pseudo segunda-ordem (Ho et al., 1996), e de difusão intrapartícula (Yang et al., 2011) foram empregados para descrever os sistemas adsorvente-adsorbato. A forma linear da equação da pseudo-primeira ordem é dada pela Equação 1.

$$
\log \left(q_{e-} q_{t}\right)=\log q_{e-}\left(K_{1} / 2,303\right) . \mathrm{t}
$$

em que $\mathrm{q}_{\mathrm{e}}$ e $\mathrm{q}_{\mathrm{t}}$ são as quantidades de corante adsorvidas $\left(\mathrm{mg} \mathrm{g}^{-1}\right)$ no equilíbrio e no tempo $\mathrm{t}$ (minutos), respectivamente; $\mathrm{k}_{1}$ é a constante de velocidade de adsorção (minutos ${ }^{-1}$ ).

O modelo linearizado de pseudo-segunda ordem pode ser representado pela Equação 2.

$$
t / q_{t}=\left(1 / K_{2} \cdot q_{e}^{2}\right)+\left(\frac{1}{q_{e}}\right) \cdot t
$$

em que $\mathrm{k}_{2}$ é a constante de velocidade de pseudo-segunda ordem $\left(\mathrm{g} \mathrm{mg}^{-1}\right.$ minutos $\left.{ }^{-1}\right), \mathrm{q}_{\mathrm{e}}$ e $\mathrm{q}_{\mathrm{t}}$ são as quantidades de corante adsorvida $\left(\mathrm{mg} \mathrm{g}^{-1}\right)$ no equilíbrio e no tempo $\mathrm{t}$ (minutos).

Alguns modelos consideram que a cinética de adsorção seja principalmente controlada pela difusão externa como: o modelo de Lagergren (pseudo-primeira ordem) e o modelo de pseudo-segunda ordem (Ho e McKay, 1999a). Tais modelos assumem que a resistência à 
transferência de massa ocorre na camada de difusão próxima à superfície adsorvente. Contudo, o mecanismo do processo de adsorção proposto por Weber e Morris (1963), considera a difusão intrapartícula um fator limitante para a transferência de massa. No modelo proposto, a taxa de agitação é considerada suficientemente alta de modo que transferência de massa intrapartícula limita a difusão. As velocidades iniciais da difusão intraparticula podem ser obtidas pela Equação 3.

$$
q_{t}=R_{i d} \cdot \sqrt{t}
$$

em que $\mathrm{q}_{\mathrm{t}}$ é a quantidade de corante sorvida em $\mathrm{mg} \mathrm{g}^{-1}$ no tempo $\mathrm{t}$ e $\mathrm{R}_{\mathrm{id}}$ é a constante de transporte intraparticula $\left(\mathrm{mg} \mathrm{g}^{-1} \mathrm{~min}^{-1 / 2}\right)$.

\subsubsection{Isotermas de adsorção}

Os ensaios foram realizados a $25{ }^{0} \mathrm{C}$ e em pH ótimo ( $\mathrm{pH}=2$ ), sendo adicionados $0,080 \mathrm{~g}$ de adsorvente em tubos de centrífuga, e $15,00 \mathrm{~mL}$ de solução dos corantes em diferentes concentrações. Posteriormente estes recipientes foram submetidos à agitação durante o tempo necessário para que o sistema atingisse o equilíbrio de adsorção, sendo posteriormente centrifugados a $3000 \mathrm{rpm}(\mathrm{F}=0,1529 \mathrm{~g})$.

A concentração de soluto adsorvido na fase sólida pode ser determinada utilizando a Equação 4.

$$
q e=\frac{\left(C_{o-} C_{e q}\right) \times W}{V}
$$

em que:

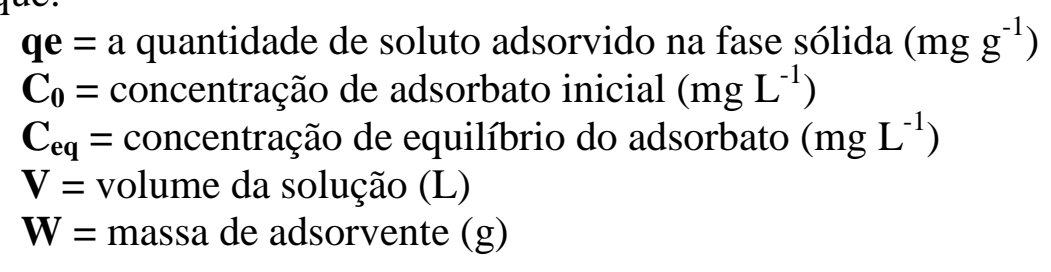

A partir dos dados podem-se obter gráficos de concentração de equilíbrio versus a quantidade adsorvida em $\mathrm{mg} \mathrm{g}^{-1}$ de adsorvente e ajustar os modelos de Langmuir e Freundlich. A isoterma de Langmuir relaciona a quantidade de soluto adsorvido em uma superfície com a concentração de equilíbrio do soluto na solução. A Equação 5 da isoterma de Langmuir é (Ho et al., 2002):

$$
q e=\frac{q_{\text {máx } \times} K_{L \times} C_{e}}{1+K_{L} \times C_{e}}
$$

em que Ce é a concentração do adsorvato na solução após o sistema atingir o equilíbrio (mg $\left.\mathrm{L}^{-1}\right), \mathrm{K}_{\mathrm{L}}$ é a constante de afinidade de Langmuir $\left(\mathrm{L} \mathrm{mg}^{-1}\right)$, $\mathrm{q}_{\text {máx }}$ é a capacidade máxima de adsorção do material $\left(\mathrm{mg} \mathrm{g}^{-1}\right)$ assumindo uma monocamada do adsorvato sobre o adsorvente.

$\mathrm{O}$ modelo de isoterma de Freundlich é uma equação exponencial não sendo possível estimar a capacidade máxima de adsorção. Teoricamente, usando a Equação 6, uma quantidade infinita de adsorção pode ocorrer.

$$
q e=K_{f \times} C_{e}^{1 / n}
$$




\subsubsection{Ensaios adsortivos em amostras de efluente têxtil}

O efluente utilizado neste trabalho foi coletado em uma tinturaria. Aos tubos de centrífuga foram adicionados $0,080 \mathrm{~g}$ de resíduos de sementes de Mabea e $15 \mathrm{~mL}$ de efluente bruto coletado. $\mathrm{O}$ efluente de cada tubo foi fortificado com cada corante separadamente, a concentração final em $30 \mathrm{mg} \mathrm{L}^{-1}$. Posteriormente este efluente teve o $\mathrm{pH}$ ajustado para o valor ótimo $(\mathrm{pH}=2)$. Os sistemas contendo os corantes $\mathrm{DR} 80$ e o RB 21 permaneceram sob agitação a $25{ }^{\circ} \mathrm{C}$ por 300 minutos e 120 minutos, respectivamente. Em seguida estes tubos foram centrifugados a $3000 \mathrm{rpm}(\mathrm{F}=0,1529 \mathrm{~g})$, durante 20 minutos. A concentração dos corantes remanescentes nos sobrenadantes foi determinada por extrapolação da curva analítica construída de $0,1,5,10$ e $15 \mathrm{mg} \mathrm{L}^{-1}$ de cada corante pelo método de adição padrão e as leituras da absorbância feitas por meio de espectrofotometria UV/VIS.

\section{RESULTADOS E DISCUSSÃO}

\subsection{Caracterização do adsorvente}

Verifica-se, de acordo com o espectro IR (Figura 1), que a posição e a forma da banda em aproximadamente $3377 \mathrm{~cm}^{-1}$ é compatível com o estiramento ( $\left.v \mathrm{O}-\mathrm{H}\right)$, referente ao grupo $\mathrm{OH}$ associado, participando de ligação de hidrogênio. A presença de fenóis é confirmada pelo estiramento C-O na faixa de $1220 \mathrm{~cm}^{-1}$ e por meio de uma deformação angular fora do plano $(\gamma \mathrm{O}-\mathrm{H})$ tipicamente alargada em $607 \mathrm{~cm}^{-1}$. Ainda destaca-se o estiramento em $3010 \mathrm{~cm}^{-1}$ referente ao estiramento $(v=\mathrm{CH})$ presente em compostos aromáticos (Silverstein e Webster, 2000). Observa-se no resíduo de semente de Mabea a presença dos grupos funcionais $\mathrm{OH}$ e anéis aromáticos que podem ser potenciais sítios de adsorção.

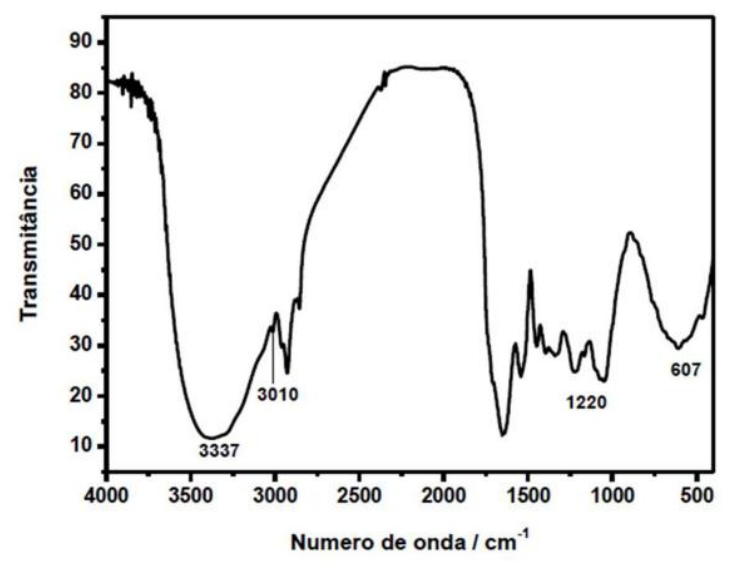

Figura 1. Espectro de IR na faixa de 400$4000 \mathrm{~cm}^{-1}$ em $\mathrm{KBr}$ referente ao resíduo de semente de Mabea fistulifera Mart.

O ponto de carga zero de um adsorvente é definido como o ponto em que a carga total da superfície é zero (neutra). Ele é um importante parâmetro usado para determinar a carga superficial dos adsorventes, uma vez que esta é dependente do $\mathrm{pH}$ da solução do adsorvato. A carga líquida na superfície do adsorvente é positiva para um $\mathrm{pH}$ da solução mais baixo que aquele correspondente ao PCZ ( $\mathrm{pH}$ PZC) e negativa para um $\mathrm{pH}$ da solução maior que o do PCZ (Moreno-Castilla, 2004). Devido à complexidade da estrutura do resíduo de semente de Mabea fistulifera Mart, o valor de PCZ (Figura 2) para este adsorvente abrange a faixa entre 4,73 e 6,00 , isto significa que nesta faixa de valores a carga total da superfície é zero, e para 
SILVEIRA NETA, J. J.; SILVA, C. J.; MOREIRA, G. C.; REIS, C.; REIS, E. L. Remoção dos corantes Reactive Blue 21 e Direct Red 80 utilizando resíduos de sementes de Mabea fistulifera Mart. como biossorvente. AmbiAgua, Taubaté, v. 7, n. 1, p. 104-119, 2012. (http://dx.doi.org/10.4136/ambi-agua.854)

valores de $\mathrm{pH}$ abaixo de 4,73 a superfície do resíduo encontra-se carregada positivamente, já para valores de $\mathrm{pH}$ acima de 6,00 a superfície se encontra carregada negativamente.

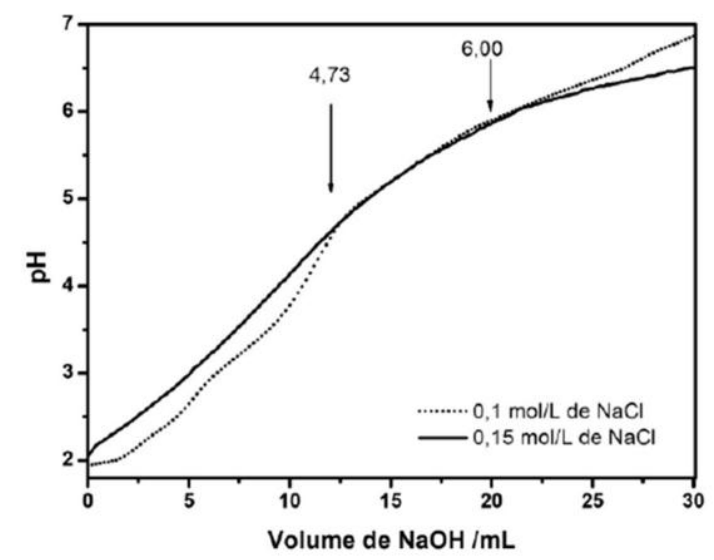

Figura 2. Curvas de titulação do resíduo de semente de Mabea fistulifera Mart. em diferentes concentrações de $\mathrm{NaCl}$

A Figura 3 mostra a superfície dos resíduos de sementes de Mabea predominantemente porosa, devido a fibras presentes em sua composição. Os poros apresentam-se com tamanhos variados, e estes contribuem para aumentar a superfície específica e, consequentemente, para reter maior quantidade de corantes. Estes resíduos foram visualizados no microscópio eletrônico de varredura obtendo-se ampliações de 667 vezes e 992 vezes.
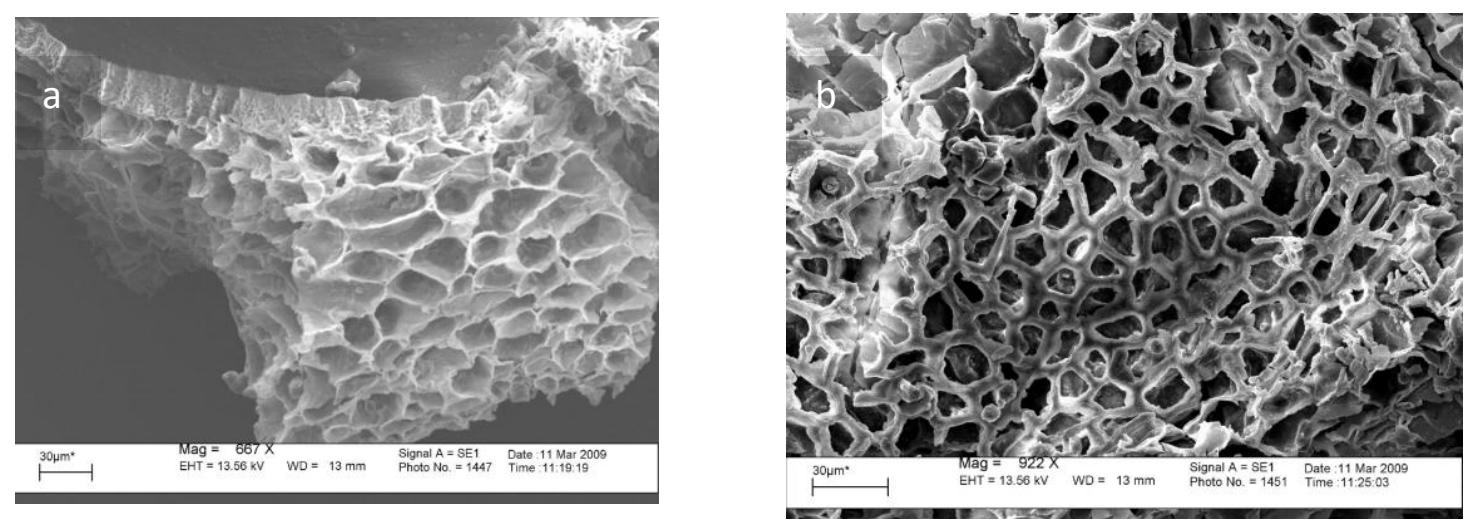

Figura 3. Micrografia da semente de Mabea in natura, com aumentos (a) 667 X (b) 992 X

\subsection{ENSAIOS ADSORTIVOS EM BATELADA}

\subsubsection{INFLUÊNCIA DO pH NA ADSORÇÃO}

A máxima adsorção referente aos corantes Direct Red 80 e Reactive Blue 21, é observada em $\mathrm{pH}$ próximo de 2, para ambos (Figura 4), para valores de $\mathrm{pH}$ superiores a 2 verifica-se uma diminuição na adsorção dos corantes. O meio ácido favorece a adsorção de ambos os corantes por resíduos de sementes de Mabea, pois a superfície do adsorvente adquire carga positiva neste meio, mas os corantes permanecem desprotonados com cargas negativas, por possuírem grupamentos com característica de ácidos fortes, proporcionando a interação. 


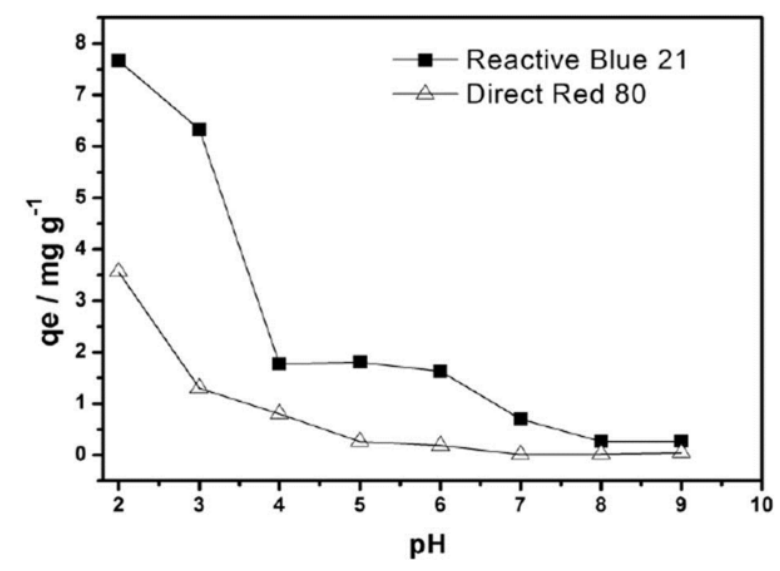

Figura 4. Variação da adsorção do corante por resíduos de sementes de Mabea em função da variação de $\mathrm{pH},(\Delta)$ Direct Red 80, (ロ) Reactive Blue $21\left(\mathrm{C}_{\mathrm{i}}=30 \mathrm{mg} \mathrm{L}^{-1}\right.$, tempo de agitação $=540 \mathrm{~min}, 0,080 \mathrm{~g}$ de adsorvente, $25^{0} \mathrm{C}$ ).

\subsubsection{ESTUDOS CINÉTICOS DE ADSORÇÃO}

As curvas cinéticas de adsorção para ambos os corantes em função do tempo (Figura 5) possibilitam verificar que a adsorção é inicialmente mais rápida e com o passar do tempo fica evidente um aumento da quantidade absorvida, até o momento em que o equilíbrio é atingido. A partir de então, não é observado mais variação da quantidade adsorvida de cada corante em função do tempo. Para DR 80, o equilíbrio é observado aos 300 minutos de agitação, e aos 120 minutos, para o RB 21, esses tempos foram utilizados na obtenção das isotermas de adsorção.

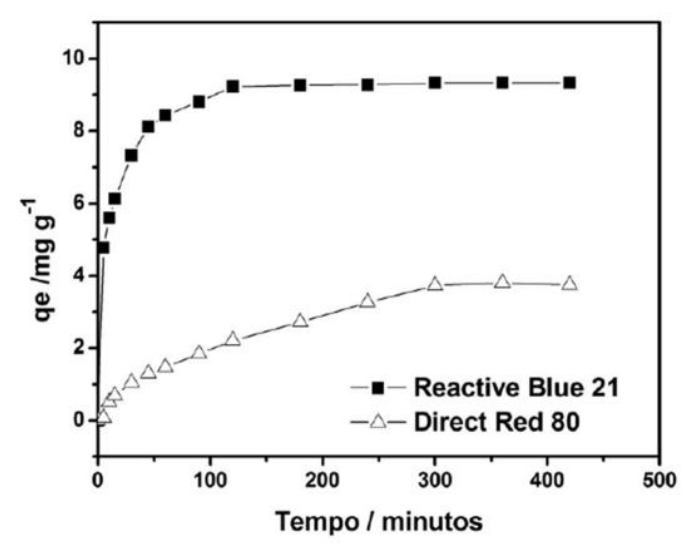

Figura 5. Variação da adsorção do corante por resíduos de sementes de Mabea em função da variação de tempo, $(\Delta)$ Direct Red 80, (घ) Reactive Blue 21 $\left(\mathrm{C}_{\mathrm{i}}=30 \mathrm{mg} \mathrm{L}^{-1}\right.$, tempo de agitação $=420$ min, $0,080 \mathrm{~g}$ de adsorvente, $25^{\circ} \mathrm{C}$ ).

Para avaliar as mudanças na taxa de adsorção dos corantes com o tempo foi necessário empregar um modelo cinético adequado. Os modelos de pseudo-primeira e pseudo-segunda ordem assumem que a diferença entre a concentração adsorvida em um determinado tempo e 
a concentração da adsorvida no equilíbrio, é a força motriz da adsorção, e a taxa de adsorção global, ou é proporcional à força motriz, no caso da equação de pseudo-primeira ordem, ou é o quadrado da força motriz, para o modelo de pseudo-segunda ordem. Estes modelos assumem ainda que a adsorção é um processo de "falsa" ordem de reação química e a taxa de adsorção pode ser determinada pelas equações de reação de primeira ordem e reação de segunda ordem (Yang e Al-Duri, 2005). Inicialmente, optou-se testar, o ajuste empregando o modelo de pseudo-primeira ordem (Figura 6), para os dados experimentais referentes ao RB 21 e ao DR 80, obtendo-se os coeficientes de determinação $r^{2}$ iguais a 0,9117 e 0,9812, respectivamente. O modelo ajustou-se melhor para a adsorção do corante DR 80. Em muitos casos, a equação de pseudo-primeira ordem não se ajusta a toda faixa de tempo de contato do adsorvato com adsorvente. Geralmente, esta é aplicável em tempos acima de 20 a 30 minutos iniciais do processo de adsorção (Ho e Mckay, 1999b) como observado para o corante RB 21 acima de 25 minutos (Figura 6). Os valores de $-\mathrm{k}_{1}$, obtidos dos coeficientes angulares da reta foram -0,07802 e -0,02210 $\mathrm{min}^{-1}$ para os corantes DR 80 e RB 21, respectivamente, e segundo Baldez et al. (2008), assumem valores negativos indicando que a concentração de solutos na solução diminuem com o aumento da tempo.

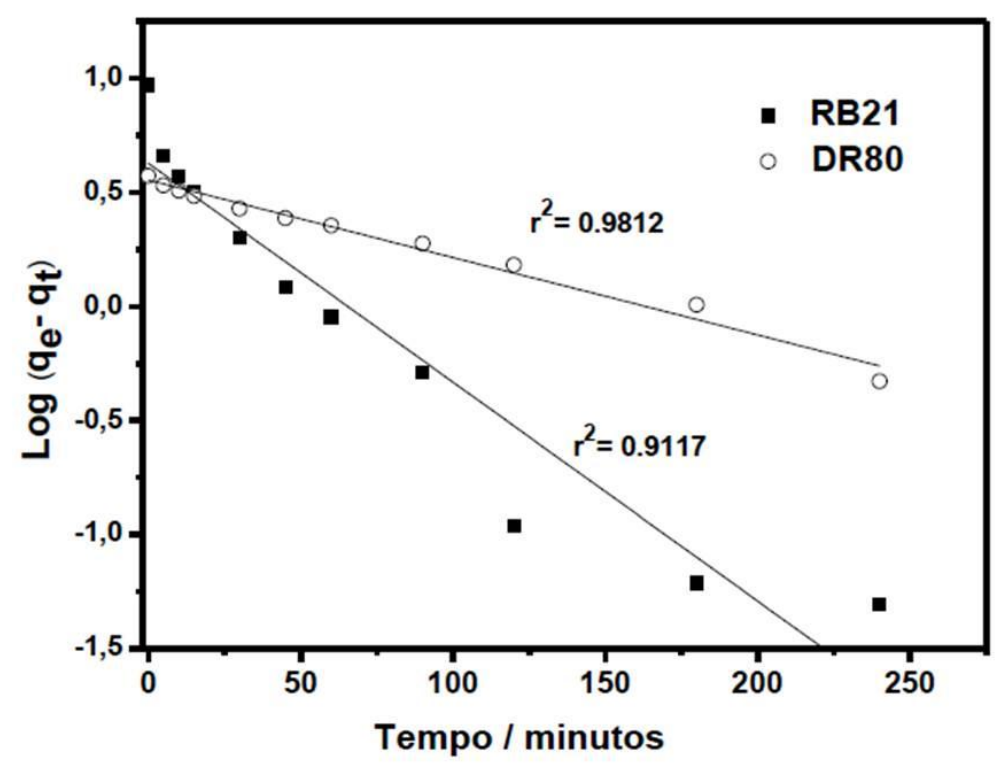

Figura 6. Aplicação do modelo de primeira- ordem para sorção do (匹) RB 21 e do (o) DR 80 pelos resíduos de Mabea Fistulifera mart. $\left(\mathrm{C}_{\mathrm{i}}=30 \mathrm{mg} \mathrm{L} \mathrm{L}^{-1}\right.$, tempo de agitação $=420$ $\min , 0,080 \mathrm{~g}$ de adsorvente, $25^{\circ} \mathrm{C}$ ).

Os valores de $\mathrm{q}_{\mathrm{e}}$ previstos pelo modelo de pseudo-segunda ordem (Figura 7) foram 3,68 $\pm 0,01 \mathrm{mg} \mathrm{g}^{-1}$ para o RB 21 e $9,53 \pm 0,03 \mathrm{mg} \mathrm{g}^{-1}$ para o DR 80, e estão em acordo com os valores de $\mathrm{q}_{\mathrm{e}}$ experimentais de $3,76 \pm 0,11 \mathrm{mg} \mathrm{g}^{-1}$ e 9,29 $\pm 0,07 \mathrm{mg} \mathrm{g}^{-1}$, respectivamente. As constantes de velocidade $\mathrm{K}_{2}$ obtidas foram $0,0017 \mathrm{~g} \mathrm{mg}^{-1}$ minutos ${ }^{-1}$ para o RB 21 e $0,0043 \mathrm{~g}$ $\mathrm{mg}^{-1}$ minutos $^{-1}$ para o DR 80. Já os coeficientes de determinação para o ajuste do modelo foram 0,9998 para RB 21 e 0, 8804 para DR 80. A cinética de pseudo-segunda ordem descreve bem processos de adsorção química, envolvendo doação ou troca de elétrons entre o adsorvato e o adsorvente, como forças covalentes e de troca iônica (Ho e Mckay, 2000), neste tipo de adsorção, as moléculas não são atraídas por todos os pontos da superfície do sólido, mas especificamente para os centros ativos, de maneira a formar uma única camada inicialmente, podendo haver a formação de outras camadas por fisissorção (Coulson and 
Richardson, 1982), O mecanismo de pseudo-segunda ordem se ajusta melhor para o RB 21, sugerindo que a quimisorção seja a forma predominante de adsorção.

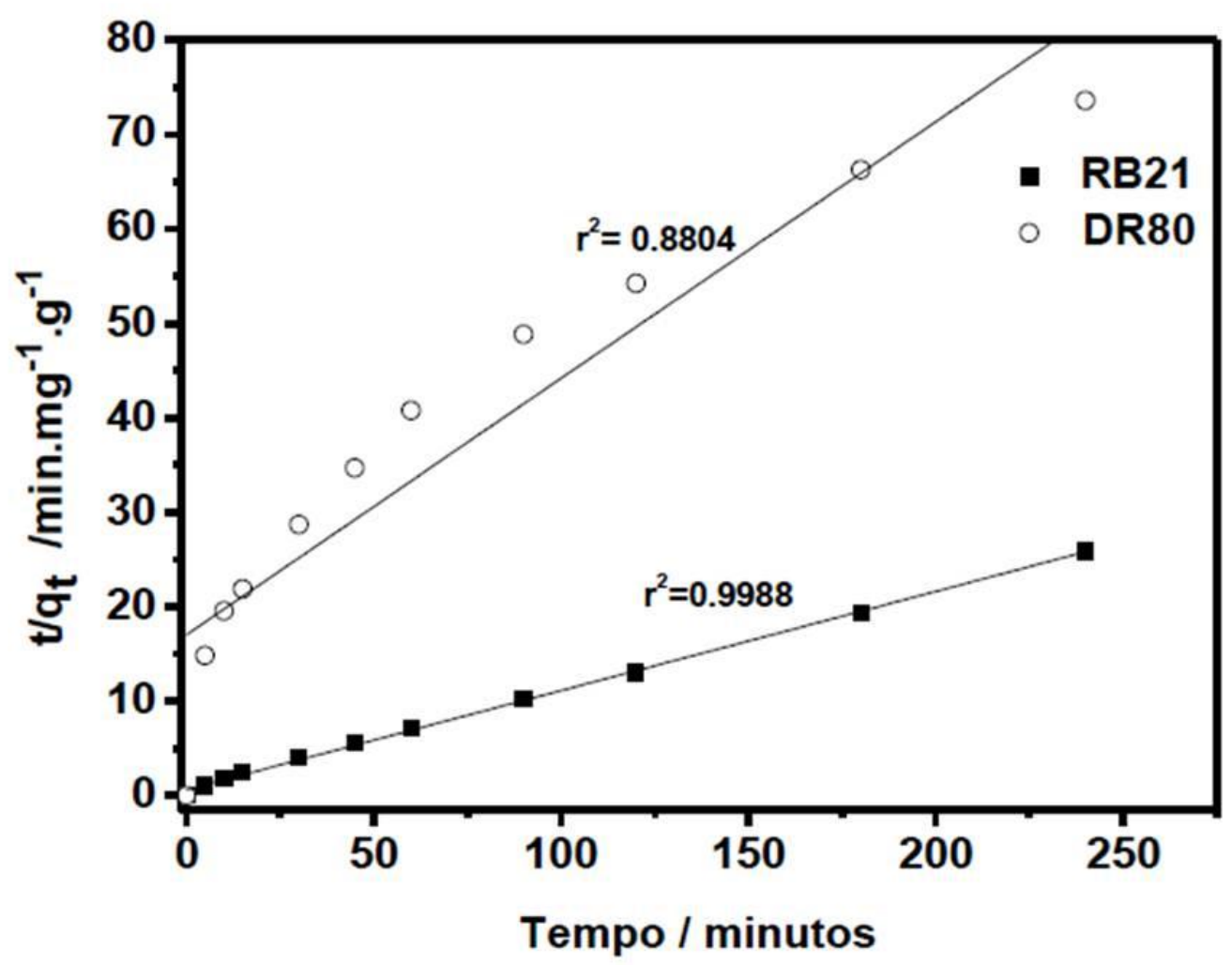

Figura 7. Aplicação do modelo de segunda- ordem para sorção do (ロ) RB 21 e do (०) DR 80 pelos resíduos de Mabea Fistulifera Mart. $\left(\mathrm{C}_{\mathrm{i}}=30 \mathrm{mg} \mathrm{L}^{-1}\right.$, tempo de agitação $=420 \mathrm{~min}, 0,080 \mathrm{~g}$ de adsorvente, $25^{\circ} \mathrm{C}$ ).

Um estudo mecanicista foi realizado com intuito de avaliar a difusão do adsorvato empregando o modelo de Morris-Weber para o sistema em estudo. Nas Figuras 8a e 8b, observa-se a aplicação deste modelo aos dados obtidos na adsorção dos corantes, obtendo-se valores de coeficiente de determinação sempre maiores que 0,9 , indicando boa correlação entre $\mathrm{q}_{\mathrm{t}}$ e $\mathrm{t}^{1 / 2}$ e mostrando que pelo menos parte do fenômeno global de sorção tem relação com a transferência intraparticula, como seria de se esperar dada a natureza porosa do material usado como adsorvente. O gráfico de quantidade adsorvida " $\mathrm{q}_{\mathrm{e}}$ versus $\mathrm{t}$ " $1 / 2$ " (Figura 8b) resulta em uma reta que passa pela origem, o que sugere que o mecanismo intra-partícula é dominante em todo processo de adsorção do corante DR 80 (Özcan et al., 2004). Enquanto que, para o corante RB 21 (Figura 8a), o gráfico não é linear durante todo o processo, sugerindo que o mecanismo de difusão intra-partícula não é dominante. Desta forma, os dados são mais bem representados por três fases lineares, sendo que a fase inicial representa o efeito de camada limite, com transferência de massa externa, em que o corante Reactive Blue 21 é rapidamente adsorvido pelos resíduos de semente de Mabea. Após 25 minutos, a velocidade da adsorção diminui, resultando na segunda fase, que se estende até 120 minutos. Esta etapa se refere à difusão das moléculas para os sítios de adsorção mais internos do adsorvente (Kumar e Porkodi, 2007). Depois de 120 minutos, observa-se o equilíbrio onde a difusão intrapartícula começa a decair devido à baixa concentração de soluto na solução bem como uma menor disponibilidade de sítios para adsorção. 

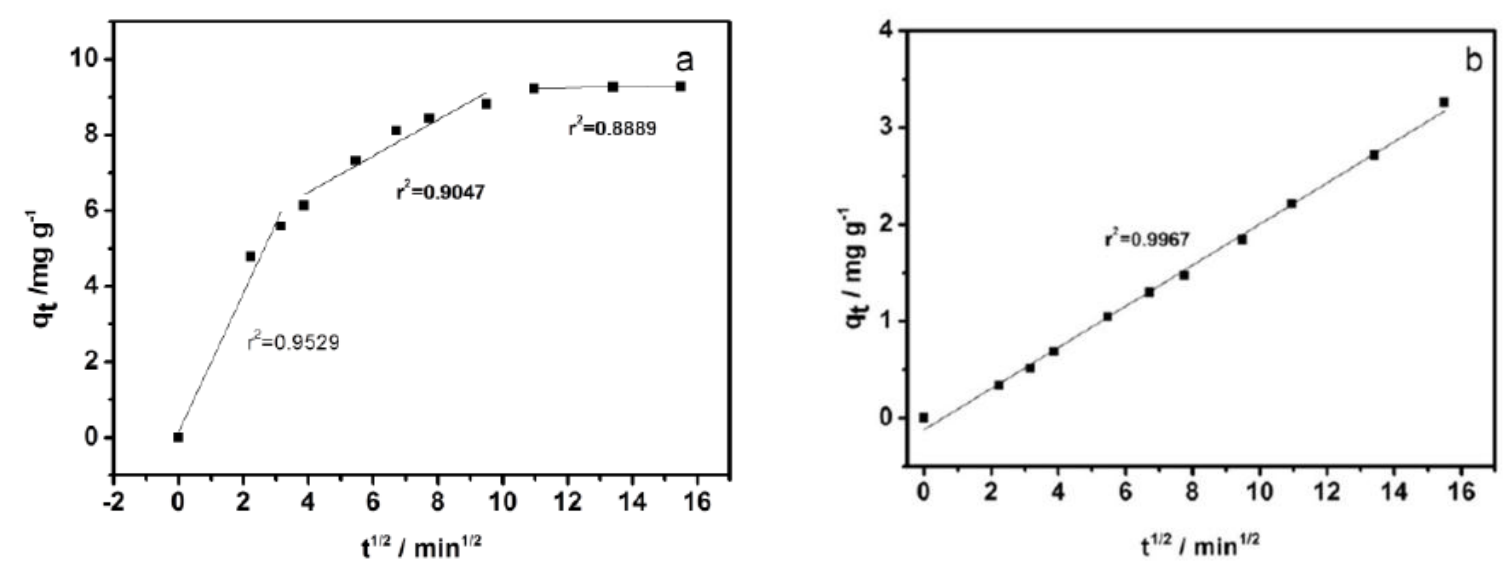

Figura 8. Aplicação do modelo de difusão intrapartícula (Morris-Weber) para sorção do (a) RB 21 e do (b) DR 80 pelos resíduos de Mabea fistulifera Mart. (Ci $=30 \mathrm{mg} \mathrm{L}^{-1}$, tempo de agitação $=420 \mathrm{~min}, 0,080 \mathrm{~g}$ de adsorvente, $25^{\circ} \mathrm{C}$ ).

\subsubsection{Isotermas de adsorção}

Estudos de adsorção dos corantes foram realizados em batelada. A partir dos dados experimentais, foi possível obter as isotermas de adsorção "qe $\left(\mathrm{mg} \mathrm{g}^{-1}\right)$ versus $C e\left(\mathrm{mg} \mathrm{L}^{-1}\right)$ " utilizando os modelos de Langmuir e Freundlich que permitiram obter os parâmetros apresentados na Tabela 2.

Tabela 2. Parâmetros obtidos do ajuste dos modelos de Langmuir e Freundlich, para ambos os corantes.

\begin{tabular}{c|ccc|ccc}
\hline \multirow{2}{*}{ Corante } & \multicolumn{3}{|c|}{ Langmuir } & \multicolumn{3}{c}{ Freundlich } \\
\cline { 2 - 7 } & $\mathbf{q}_{\text {máx }}\left(\mathbf{m g ~ g}^{-\mathbf{1}}\right)$ & $\mathbf{K}_{\mathbf{L}}$ & $\mathbf{r}^{\mathbf{2}}$ & $\mathbf{K}_{\mathbf{f}}$ & $\mathbf{n}$ & $\mathbf{r}^{\mathbf{2}}$ \\
\hline DR 80 & $4,92 \pm 0,27$ & $0,03 \pm 0,00$ & 0,9812 & $0,53 \pm 0,08$ & $2,42 \pm 0,03$ & 0,9794 \\
\hline RB 21 & $11,13 \pm 0,73$ & $0,02 \pm 0,00$ & 0,9895 & $0,68 \pm 0,18$ & $1,96 \pm 0,05$ & 0,9621 \\
\hline
\end{tabular}

O modelo de Langmuir (Figura 9 a e Tabela 2) apresentou melhor ajuste $\left(R^{2}>0,98\right)$ quando comparado ao ajuste do modelo de Freundlich para ambos os corantes.

A capacidade máxima de adsorção foi estimada pelo modelo de Langmuir, sendo de 4,91 $\mathrm{mg} \mathrm{g}^{-1}$ para DR 80 , e $11,13 \mathrm{mg} \mathrm{g}^{-1}$ para $\mathrm{RB} 21$, resultados superiores aos encontrados em outro estudo que trata da adsorção dos corantes RB 21 e DR 80 por espumas de poliuretano (Neta et al., 2011), e para adsorção de azul de metileno por caulinita (Leal et al., 2011).

Para ambos, o processo de adsorção se mostrou favorável, visto que a faixa de variação do valor de RL foi de 0,14 a 0,82 para o DR 80 e de 0,21 a 0,87 para o RB 21. Quando o valor de RL varia entre 0 e 1, significa que a adsorção é favorável (Bhattacharyya e Sarma, 2003).

A partir das isotermas ajustadas pelo modelo Freundlich (Figura 9 b e Tabela 2), foi possível obter os valores da constante de Freundlich (n) para o corante DR 80 é 2,42 e para o corante RB 21 é 1,96, representando também condições de adsorção (Fritz e Schlünder, 1981). 

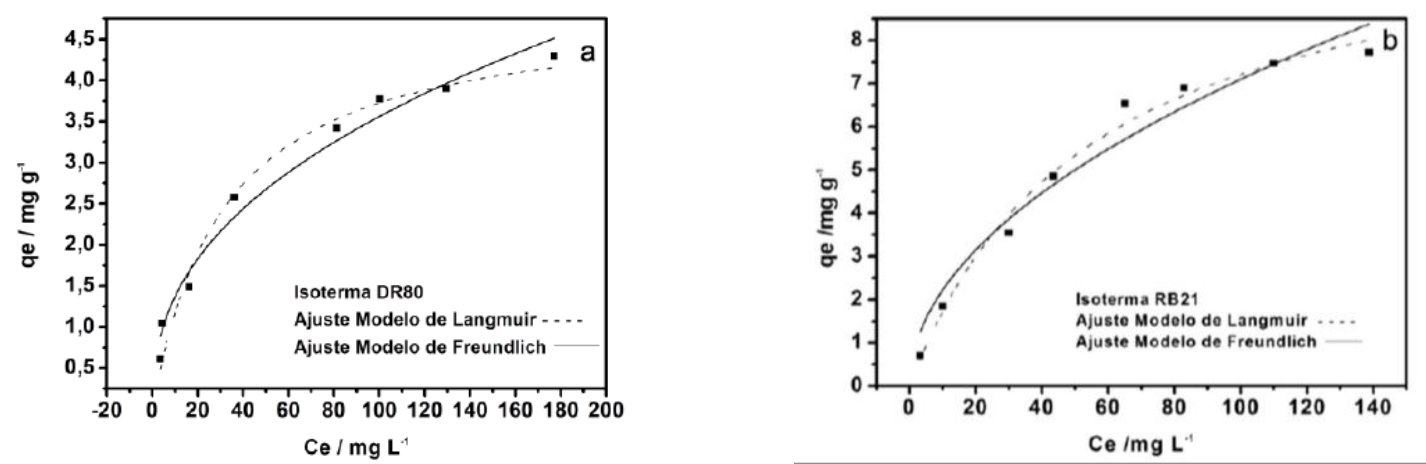

Figura 9. Ajuste da isoterma de Langmuir e Freundlich aos dados da adsorção (a) Direct Red 80, pH $=2$ e tempo de agitação 300 minutos, (b) Reactive Blue 21, $\mathrm{pH}=2$ e tempo de agitação 120 minutos. $\left(\mathrm{Ci}=30 \mathrm{mg} \mathrm{L}^{-1}, 0,080 \mathrm{~g}\right.$ de adsorvente, $\left.25^{\circ} \mathrm{C}\right)$.

\subsubsection{Ensaios adsortivos em amostras de efluente têxtil}

Com o intuito de testar a possibilidade de aplicação da técnica desenvolvida neste trabalho em amostras reais de efluentes industriais, um experimento foi realizado para a remoção de DR 80 e RB 21 de um efluente fortificado com os corantes. Tal teste foi realizado individualmente para cada corante estudado.

As condições experimentais empregadas foram àquelas estabelecidas ao longo do desenvolvimento do trabalho, sendo possível, alcançar uma recuperação de $94 \%$ do corante RB 21 e $85 \%$ para o corante DR 80.

A evolução da remoção dos corantes RB 21 e DR 80 é apresentada na Figura 10, que mostra os espectros registrados durante procedimento de adsorção. A redução acentuada da intensidade da banda de máxima absorção em $537 \mathrm{~nm}$ para o corante Direct Red 80, e em 631 $\mathrm{nm}$ para o corante Reactive Blue 21 foi observada, evidenciando que os corantes foram removidos dos efluentes por ação dos resíduos de sementes de Mabea fistulifera Mart.

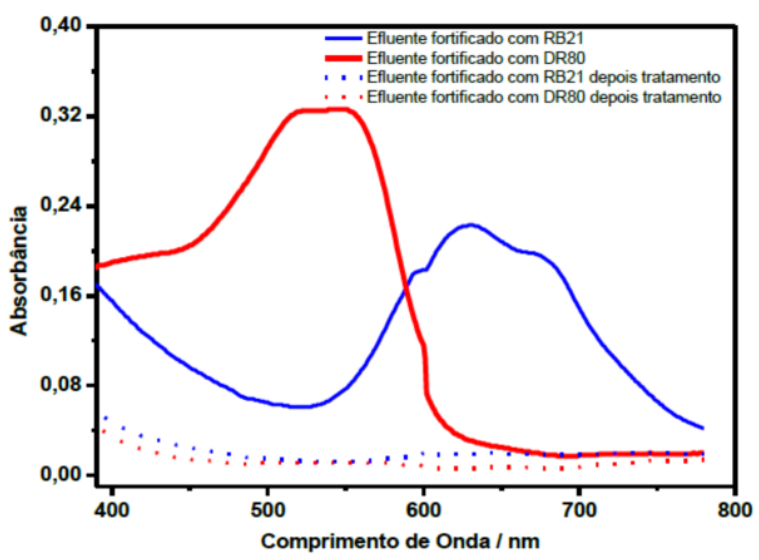

Figura 10. Espectros obtidos de amostra de efluente têxtil fortificado: antes da remoção dos corantes RB 21( - ) e DR 80( - ) e depois da remoção de corantes RB 21 (...) e DR 80 (...). 


\section{CONCLUSÃO}

O estudo da adsorção em batelada dos corantes Direct Red 80 e Reactive Blue 21 por resíduos de Mabea fistulifera Mart. revelou que o processo é fortemente afetado pelo $\mathrm{pH}$, sendo favorecido em meio ácido. A máxima adsorção foi observada em $\mathrm{pH}=2$ para ambos os corantes, neste valor de $\mathrm{pH}$ a superfície do adsorvente se encontra carregada predominantemente com cargas positivas $(\mathrm{pH}<\mathrm{pH}$ PCZ) que interagem com grupamentos das moléculas de corantes carregados negativamente. O tempo de equilíbrio do sistema adsortivo foi estabelecido em 120 minutos para o corante RB21 e 300 minutos para o corante DR80. O modelo de Langmuir se mostrou mais adequado para descrever os sistemas corantesadsorvente, e a capacidade máxima encontrada utilizando este modelo foi de $4,91 \mathrm{mg} \mathrm{g}^{-1}$ para o DR80 e $11,13 \mathrm{mg} \mathrm{g}^{-1}$ para o RB21 em condições de $\mathrm{pH}=2$ e a $25{ }^{0} \mathrm{C}$. O modelo cinético de pseudo-segunda ordem melhor descreveu o processo de adsorção do corante RB21 sob o bioadsorvente, embora a difusão intra-partícula também esteja envolvida no mecanismo de adsorção. Já para o corante DR80 o modelo de difusão de Morris e Weber sugeriu que a difusão intra-partícula é predominante em todo o processo de adsorção.

Depois de estabelecida as condições adsortivas empregando apenas soluções aquosas dos corantes, o processo de tratamento desenvolvido foi aplicado a uma amostra real de efluente industrial fortificado com soluções de $30 \mathrm{mg} \mathrm{L}^{-1}$ de cada corante separadamente. Foi observado um percentual de remoção de $94 \%$ para o RB21 e $85 \%$ para o DR80 empregando os resíduos de Mabea como adsorvente dos corantes presentes no efluente industrial.

Os resíduos de Mabea fistulifera Mart. revelaram elevado potencial para aplicação como bioadsorvente principalmente em efluentes indústrias ácidos, que apresentem em sua constituição os corantes Direct Red 80 e Reactive Blue 21.

\section{AGRADECIMENTOS}

À CAPES (Coordenação de Aperfeiçoamento pessoal de Nível Superior) pelo apoio financeiro.

\section{REFERÊNCIAS}

ACHAK, M.; HAFIDI, A.; OUAZZANI, N.; SAYADI, S.; MANDI, L. Low cost biosorbent "banana peel" for the removal of phenolic compounds from olive mill wastewater: kinetic and equilibrium studies. Journal of Hazardous Materials, v. 166, n. 1, p. 117125, 2009. http://dx.doi.org/10.1016/j.jhazmat.2008.11.036

AKTAŞ, O.; ÇEÇEN, F. Adsorption, desorption and bioregeneration in the treatment of 2chlorophenol with activated carbon. Journal of Hazardous Materials, v. 141, n. 3, p. 769-777, 2007. http://dx.doi.org/10.1016/j.jhazmat.2006.07.050

BAEK, M.; IJAGBEMI, C. O.; SE-JIN, O.; KIM, D. Removal of Malachite Green from aqueous solution using degreased coffee bean. Journal of Hazardous Materials, v. 176, n. 1-3, p. 820-828, 2010. http://dx.doi.org/10.1016/j.jhazmat.2009.11.110

BALDEZ, E. E.; ROBAINA, N. F.; CASSELLA, R. J. Employment of polyurethane foam for the adsorption of Methylene Blue in aqueous. Journal of Hazardous Materials, v. 159, n. 2-3, p. 580-586, 2008. http://dx.doi.org/10.1016/j.jhazmat.2008.02.055

BHATTACHARYYA, K. G.; SARMA, A. Adsorption characteristics of the dye, Brilliant Green, on Neem leaf powder. Dyes Pigments, v. 57, n. 3, p. 211-222, 2003. http://dx.doi.org/10.1016/S0143-7208(03)00009-3 
BRITO, S. M. O.; ANDRADE, H. M. C.; SOARESA, L. F.; AZEVEDO, R. P. Brazil nut shells as a new biosorbent to remove methylene blue and indigo carmine from aqueous solutions. Journal of Hazardous Materials, v. 174, n. 1-3, p. 84-92, 2010. http://dx.doi.org/10.1016/j.jhazmat.2009.09.020

CHEN, G. Q.; ZHANG, W. J.; ZENG, G. M.; HUANG, J. H.; WANG, L.; SHEN, G. L. Surface-modified Phanerochaete chrysosporium as a biosorbent for $\mathrm{Cr}$ (VI)contaminated wastewater. Journal of Hazardous Materials, v. 186, n. 2-3, p. 2138 2143, 2011. http://dx.doi.org/10.1016/j.jhazmat.2010.12.123

COULSON, J. M.; RICHARDSON, J. F. Tecnologia química, Lisboa: Fundação Calouste Gulbenkian, 1982. 746 p.

EGREJA FILHO, F. B; TEÓFILO, R. F.; REIS, E. L.; REIS, C.; FONTES, M. P. F. Determinação do ponto de carga zero por efeito salino (PCZES) em amostras de solos, por titulação contínua. Revista Tecno-Lógica, UNISC, v. 8, n. 2, p. 51-59, 2004.

FRITZ, W.; SCHLÜNDER, E. U. Competitive adsorption of two dissolved organics onto activated carbon - I Adsorption Equilibria. Chemical Engineering Science, v. 36, n. 4, p. 721-730, 1981. http://dx.doi.org/10.1016/0009-2509(81)85088-9

FROEHNER, S.; MARTINS, R. F.; FURUKAWA, W.; ERRERA, M. R. Water remediation by adsorption of phenol onto hydrophobic modified clay. Water, Air \& Soil Pollution, v. 199, n. 1-4, p. 107-113, 2009. http://dx.doi.org/10.1007/s11270-008-9863-0

GHORBANI, F.; YOUNESI, H.; GHASEMPOURI, S. M.; ZINATIZADEH, A. A.; AMINI, M.; DANESHI, A. Application of response surface methodology for optimization of cadmium biosorption in an aqueous solution by Saccharomyces cerevisiae. Chemical Engineering Journal, v. 145, n. 2, p. 267-275, 2008. http://dx.doi.org/10.1016/ j.cej.2008.04.028

HO, Y. S.; HUANG, C. T.; HUANG, H. W. Equilibrium sorption isotherm for metal ions on tree fern. Process Biochemistry, v. 37, n. 12, p. 1421-1430, 2002. http://dx.doi.org/10.1016/S0032-9592(02)00036-5

HO, Y. S; WASE, D. A. J.; FORSTER, C. F. Kinetic studies of competitive heavy metal adsorption by sphagnum moss peat. Environmental Technology, v. 17, n. 1, p. 71-77, 1996. http://dx.doi.org/10.1080/09593331708616362

HO, Y. S.; MCKAY, G. Pseudo-second order model for sorption processes. Process Biochemistry, v. 34, n. 5, p. 451-465, 1999a. http://dx.doi.org/10.1016/S00329592(98)00112-5

HO, Y. S.; MCKAY, G. The kinetics of sorption of divalent metal ions ontos phagnum moss peat. Water Research, v. 34, n. 3, p. 735-742, 2000. http://dx.doi.org/10.1016/S00431354(99)00232-8

HO. Y. S.; MCKAY, G. The Sorption of Lead (II) Ions on Peat. Water Research, v. 33, n. 2, p. 578-584, 1999b. http://dx.doi.org/10.1016/S0043-1354(98)00207-3

IMMICH, A. P. S.; SOUZA, A. A. U; SOUZA, S. M. A. G. U. Removal of Remazol Blue RR dye from aqueous solutions with Neem leaves and evaluation of their acute toxicity with Daphnia magna. Journal of Hazardous Materials, v. 164, n. 2-3, p. 1580-1585, 2009. http://dx.doi.org/10.1016/j.jhazmat.2008.09.019 
KESKINKAN, O.; GOKSU, M. Z. L.; BASIBUYUK, M.; FORSTER, C. F. Heavy metal adsorption properties of a submerged aquatic plant (Ceratophyllum demersum).

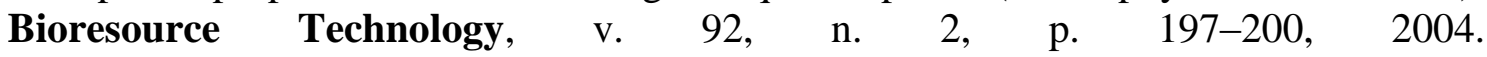
http://dx.doi.org/10.1016/j.biortech.2003.07.011

KUMAR, K. V.; PORKODI, K. Mass transfer, kinetics and equilibrium studies for the biosorption of methylene blue using paspalum notatum. Journal of Hazardous Materials, v. 146, n. 1-2, p. 214-226, 2007. http://dx.doi.org/10.1016/j.jhazmat.2006.12.010

LEAL, P. V. B.; DOS ANJOS, J. P.; MAGRIOTIS, Z. M. Estudo da adsorção do corante azul de metileno em caulinita rosa. Journal of Biotechnology and Biodiversity, v. 2, n. 3, p. 38-42, 2011.

LOHANI, M. B.; SINGH, A.; RUPAINWAR, D. C.; DHAR, D. N. Studies on efficiency of guava (Psidium guajava) bark as bioadsorbent for removal of $\mathrm{Hg}(\mathrm{II})$ from aqueous solutions. Journal of Hazardous Materials, v. 159, n. 2-3, p. 626-629, 2008. http://dx.doi.org/10.1016/j.jhazmat.2008.02.072

LORENZI, H. As árvores brasileiras: manual de identificação e cultivo de plantas arbóreas nativas do Brasil. Nova Odessa: Plantarum, 2000. 322 p.

MORENO-CASTILLA, C. Adsorption of organic molecules from aqueous solutions on carbon materials. Carbon, v. 42, n. 1, p. 83-94, 2004.

http://dx.doi.org/10.1016/j.carbon.2003.09.022

SILVEIRA NETA, J. de J.; MOREIRA, G. C.; DA SILVA, C. J.; REIS, C.; REIS, E. L. Use of polyurethane foams for the removal of the Direct Red 80 and Reactive Blue 21 dyes in aqueous medium. Desalination, v. 281, p. 55-60, 2011.

http://dx.doi.org/10.1016/j.desal.2011.07.041

ÖZCAN, A. SAFA; ÖZCAN, ADNAN. Adsorption of Acid Dyes from Aqueous Solutions Onto Acid-Activated Bentonite. Journal of Colloid and Interface Science, v. 276, n. 1, p. 39-46, 2004. http://dx.doi.org/10.1016/j.jcis.2004.03.043

OZKAYA, B. Adsorption and desorption of phenol on activated carbon and a comparison of isotherm models. Journal of Hazardous Materials, v. B 129, n. 1-3, p. 158-163, 2006. http://dx.doi.org/10.1016/j.jhazmat.2005.08.025

PARK, C.; LEE, M.; LEE, B.; KIM, SW.; CHASE, H.A.; LEE, J.; KIM, S. Biodegradation and biosorption for decolorization of synthetic dyes by Funalia trogii. Biochemical Engineering Journal, v. 36, n. 1, p. 59-65, 2007.

http://dx.doi.org/10.1016/j.bej.2006.06.007

PARSHETTI, G. K.; TELKE, A. A.; KALYANI, D. C.; GOVINDWAR, S. P. Decolorization and detoxification of sulfonated azo dye methyl orange by Kocuria rosea MTCC 1532. Journal of Hazardous Materials, v. 176, n. 1-3, p. 503-509, 2010. http://dx.doi.org/10.1016/j.jhazmat.2009.11.058

PEREIRA, F. E. A. Biodiesel produzido a partir do óleo de sementes de Mabea fistulifera Mart. 2007. 87f. Dissertação (Mestrado em Agroquímica) - Universidade Federal de Viçosa, Viçosa, MG, 2007. 
ROBINSON, T.; CHANDRAN, B.; NAIDU, S. G.; NIGAM, P. Studies on the removal of dyes from a synthetic textile effluent using barley husk in static-batch mode and in a continuous flow, packed-bed, reactor. Bioresource Technology, v. 85, n. 1, p. 43-49, 2002. http://dx.doi.org/10.1016/j.jhazmat.2009.11.058

SAHA, P. Assessment on the removal of methylene blue dye using tamarind fruit shell as biosorbent. Water, Air \& Soil Pollution, v. 213, n. 1-4, p. 287-299, 2010. http://dx.doi.org/10.1007/s11270-010-0384-2

SANTOS, V. C. G.; SOUZA, J. V. T. M.; TARLEY, C. R. T.; CAETANO, J.; DRAGUNSKI, D. C. Copper ions adsorption from aqueous medium using the biosorbent sugarcane bagasse in natura and chemically modified. Water, Air \& Soil Pollution, v. 216, n. 1-4, p. 351-359, 2011. http://dx.doi.org/10.1007/s11270-010-05373

VIEIRA, A. M. S.; VIEIRA, M. F.; SILVA, G. F.; ARAÚJO, A. A.; FAGUNDES-KLEN, M. R.; VEIT M. T. et al. Use of moringa oleifera seed as a natural adsorbent for wastewater treatment. Water, Air \& Soil Pollution, v. 206, n. 1, p. 273-281, 2009. http://dx.doi.org/10.1007/s11270-009-0104-y

WEBER, W. J.; MORRIS, J.C. Kinetics of adsorption carbon from solutions. Journal of the Sanitary Engineering Division, v. 89, n. 2, p. 31-60, 1963.

WENG, C. H.; LIN, Y. T.; TZENG, T. W. Removal of methylene blue from aqueous solution by adsorption onto pineapple leaf powder. Journal of Hazardous Materials, v. 170, n. 1, p. 417-424, 2009. http://dx.doi.org/10.1016/j.jhazmat.2009.04.080

YANG, X; AL-DURI, B. Kinetic modeling of liquid-phase adsorption of reactive dyes on activated carbon. Journal of Colloid and Interface Science, v. 287, n. 1, p. 25-34, 2005. http://dx.doi.org/10.1016/j.jcis.2005.01.093

YANG, Y.; WANG, G.; WANG, B.; LI, Z.; JI, X.; ZHOU, Q.; ZHAO, Y. Biosorption of Acid Black 172 and Congo Red from aqueous solution by nonviable Penicillium YW 01: kinetic study, equilibrium isotherm and artificial neural network modeling. Bioresource Technology, v. 102, n. 2, p. 828-834, 2011. http://dx.doi.org/10.1016/j.biortech.2010.08.125

ZANONI, M. V. B.; CARNEIRO, P. A. O descarte dos corantes têxteis. Revista Ciência Hoje, v. 29, n. 174, p. 61-64, 2001. 